\title{
SOME APPLICATIONS OF THE TRACE MAPPING FOR DIFFERENTIALS
}

\author{
MASUMI KERSKEN and UWE STORCH \\ Ruhr-Universität Bochum, Fakultät für Mathematik, Bochum, F.R.G.
}

In memoriam Erich Platte

In this paper we study the trace mapping for differentials of (complex) analytic algebras, which has already been used in [4] and [5]. We give new proofs of essential results in the papers [17], [18], [19] and [20] by E. Platte, moreover we generalize some of these results.

The base field is assumed to be the field $\mathbf{C}$ of complex numbers only for clearness and simplicity. But it is important to notice that the characteristic of the field is zero.

(1) Notations. By a complex analytic algebra $A$ we understand a residue algebra of a formal or convergent power series algebra $\mathbf{C}\left\langle\left\langle X_{1}, \ldots, X_{n}\right\rangle\right\rangle$ over C. In the following $A$ is additionally assumed to be reduced and pure dimensional. $\Omega_{A}^{*}=\left(\Omega_{A}^{*}, d\right)$ denotes the complex of Kähler or holomorphic differentials, and $M_{A}^{*}=Q(A) \otimes_{A} \Omega_{A}^{*}=\left(M_{A}^{*}, d\right)$ denotes the complex of meromorphic differentials. $\Omega_{A}^{*}$ is the exterior algebra of the universally finite differential module $D_{\mathbf{C}}(A)=\Omega_{A}^{1}$ of $A, M_{A}^{*}$ is the exterior algebra of the free module $Q(A) \otimes_{A} \Omega_{A}^{1}=M_{A}^{1}$ over the total quotient ring $Q(A)$ of $A$. The rank of $\Omega_{A}^{1}$, i.e. the rank of $M_{A}^{1}$ is $\operatorname{dim} A$. See also [13] and [23].

The kernel of the canonical complex homomorphism $\Omega_{A}^{*} \rightarrow M_{A}^{*}$ is the torsion subcomplex $\mathrm{t} \Omega_{A}^{*}$ of $\Omega_{A}^{*}$. We always identify $\widetilde{\Omega}_{A}^{*}:=\Omega_{A}^{*} / t \Omega_{A}^{*}$ with its image in $M_{A}^{*}$ and hence as a subcomplex and a subalgebra of $M_{A}^{*}$.

(2) The trace mapping. Let $A \rightarrow B$ be a finite extension of reduced pure-d-dimensional analytic $\mathbf{C}$-algebras with a well defined rank $r$, i.e. $Q(B)$ is a free extension of $Q(A)$ with rank $r$. Then $M_{B}^{*}=Q(B) \otimes_{Q(A)} M_{A}^{*}$, and the trace mapping Sp: $Q(B) \rightarrow Q(A)$ induces an $M_{A}^{*}$-linear trace mapping $\mathrm{Sp} \otimes_{Q(A)} M_{A}^{*}$

This paper is in final form and no version of it will be submitted for publication elsewhere. 
from $M_{B}^{*}$ to $M_{A}^{*}$, which we also denote by

$$
\mathrm{Sp}_{A}^{B}: M_{B}^{*} \rightarrow M_{A}^{*} \text {. }
$$

Obviously $\mathrm{Sp}_{A}^{B} \mid M_{A}^{*}=r \mathrm{id}_{M_{A}^{*}}$. This trace mapping commutes with the exterior derivatives $d$ on $M_{A}^{*}$ and $M_{B}^{*}$. For this it is sufficient to verify that the diagram

$$
\begin{aligned}
& Q(B) \stackrel{d}{\rightarrow} M_{B}^{1} \\
& \mathrm{Sp} \downarrow \\
& Q(A) \stackrel{d}{\rightarrow} M_{A}^{1}
\end{aligned}
$$

is commutative. This may be done explicitly for elements $y \in Q(B)$ by using representations $y x_{\sigma}=\sum_{e} a_{\rho \sigma} x_{e}, d x_{\sigma}=\sum_{Q} \omega_{\rho \sigma} x_{e}$ with a $Q(A)$-base $x_{1}, \ldots, x_{r}$ of $Q(B)$ and coefficients $a_{\rho \sigma} \in Q(A), \omega_{\rho \sigma} \in M_{A}^{1}$ (see also [5] and the more general discussions in [7], $\S 4$ and [15], $\S 4)$. In the convergent case one can use alternatively a finite (ramified) covering $Y \rightarrow X$ of complex space germs which represents the extension $A \rightarrow B$. For a given $\omega \in M\}$ one removes the ramification points of the covering and the singular points of $\omega$, the problem is then reduced to the trivial case of a holomorphic form and a finite unramified covering.

We remark that for $p \in \mathbf{Z}$ the canonical diagram

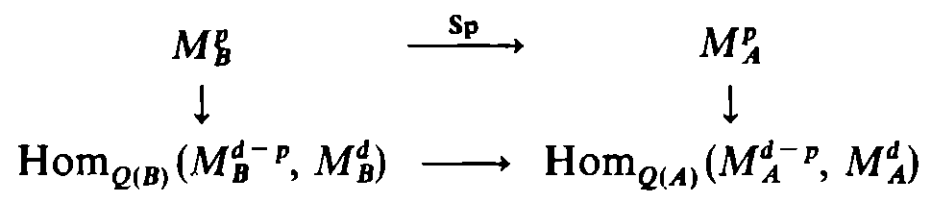

is commutative, where the homomorphism of the bottom row is the composition of the trace mapping on $M_{B}^{d}$ and the restriction to $M_{A}^{d-p}$.

As a first application of the existence of the trace mapping we prove the following result which answers a question raised by G. Scheja and has been proved for the first time by E. Platte.

(3) Proposition (Platte [18]). Let $P$ : $=\mathbf{C}\left\langle\left\langle X_{1}, \ldots, X_{n}\right\rangle\right\rangle$ and $f \in \mathbf{m}_{p}^{2}$ a function which defines an isolated singularity. Then the degree of $f$ over $Q:=\mathrm{C}\left\langle\left\langle\partial f / \partial X_{1}, \ldots, \partial f / \partial X_{n}\right\rangle\right\rangle$ equals the rank of $P$ over $Q$ and has hence the maximal value.

Proof. Let $\partial_{i}:=\partial / \partial X_{i}=\left(d X_{i}\right)^{*}$ be the partial derivations and $A$ the normalization of the hypersurface algebra

$$
Q\langle\langle f\rangle\rangle=Q[f]=\mathrm{C}\left\langle\left\langle\partial_{1} f, \ldots, \partial_{n} f, f\right\rangle\right\rangle .
$$

We have to show that $P=A$. From

$$
\omega_{i}:=\operatorname{Sp}_{A}^{B}\left(d X_{i}\right)=d \operatorname{Sp}_{A}^{B}\left(X_{i}\right) \in \widetilde{\Omega}_{A}^{1}=\Omega_{A}^{1} / \mathrm{t} \Omega_{A}^{1} \subseteq M_{A}^{1}
$$

and the equality $d f=\sum_{i} \partial_{i} f d X_{i} \in \tilde{\Omega}_{A}^{1} \subseteq \Omega_{P}^{1}$ we get with the trace mapping

$$
r d f=\sum_{i}\left(\partial_{i} f\right) \omega_{i} \in \widetilde{\Omega}_{A}^{1} \subseteq \Omega_{P}^{1},
$$


where $r \geqslant 1$ is the rank of $P$ over $A$. Applying the linear forms $\partial_{j}$ we get

$$
r \partial_{j} f=\sum_{i}\left(\partial_{i} f\right)\left(\partial_{j} \omega_{i}\right)
$$

Since $\partial_{1} f, \ldots, \partial_{n} f$ generate minimally the $P$-ideal $\sum_{j} P \partial_{j} f$ the matrix $(1 / r)\left(\partial_{j} \omega_{i}\right)$ concides modulo $m_{p}$ with the identity matrix. In particular $\left(\partial_{j} \omega_{i}\right)$ is invertible and $\omega_{1}, \ldots, \omega_{n}$ is a $P$-base of $\Omega_{P}^{1}$. Therefore $P$ is an unramified finite extension of $A$, and this means $P=A$.

We remark that in the proof of Proposition (3) besides the finiteness of $P$ over $A$ we only used the facts that $A$ is normal and contains the function $f$ together with its partial derivatives $\partial_{1} f, \ldots, \partial_{n} f$ and that $\partial_{1} f, \ldots, \partial_{n} f$ generate minimally a $P$-ideal. The last condition means (by Zariski's lemma, see [14]), that there is no coordinate system $\tilde{X}_{1}, \ldots, \tilde{X}_{n}$ for $P$, such that $f$ is independent of one of the functions $\tilde{X}_{i}$.

In the situation of Proposition (3) the rank of $P$ over $Q$, i.e. the degree of $f$ over $Q$ is equal to the Milnor number $\mu$ of the singularity $P / P f$. The Jacobian of the extension $Q \rightarrow P$ is the Hesse determinant Hesse $(f)=\operatorname{Det}\left(\partial^{2} f / \partial X_{i} \partial X_{j}\right)$. From a representation $\partial f / \partial X_{j}=\sum_{i} a_{i j} X_{i}$ with $a_{i j} \in P$ one gets

$$
\operatorname{Hesse}(f)=\mu \operatorname{Det}\left(a_{i j}\right) \bmod \left(\partial f / \partial X_{1}, \ldots, \partial f / \partial X_{n}\right),
$$

cf. [24]. Furthermore we mention that the minimal equation of the function $f$ over $\mathbf{C}\left\langle\left\langle\partial_{1} f, \ldots, \partial_{n} f\right\rangle\right\rangle$ is also an equation of integral dependence of $f$ over the ideal $\sum_{j}\left(\partial_{j} f\right) P$ generated by its partial derivatives, cf. [22].

(4) Regular and extendable differential forms. In general the trace $\mathrm{Sp}_{A}^{B}$ : $M_{B}^{*} \rightarrow M_{A}^{*}$ doesn't map $\tilde{\Omega}_{B}^{*}=\Omega_{B}^{*} / t \Omega_{B}^{*}$ into $\tilde{\Omega}_{A}^{*}=\Omega_{A}^{*} / t \Omega_{A}^{*}$. But the complexes of regular resp. extendable differential forms which we shall define now are invariant under the trace mapping. $\left(\mathrm{Sp}\left(\widetilde{\Omega}_{B}^{*}\right) \subseteq \widetilde{\Omega}_{A}{ }^{\circ}\right.$ holds in the special case, that $A \rightarrow B$ is a finite free extension, cf. for example [7], $\S 4$.)

Let $A$ be pure-d-dimensional and reduced and

$$
P \rightarrow A, P:=\mathrm{C}\left\langle\left\langle X_{1}, \ldots, X_{d}\right\rangle\right\rangle
$$

a noetherian normalization of $A$. Then we get a subcomplex $\Delta_{A}{ }^{\circ}=\left(\Delta_{A}^{*}, d\right)$ of $\left(M_{A}^{*}, d\right)$ by setting

$$
\Delta_{A}^{i}:=\left\{\omega \in M_{A}^{i}: \operatorname{Sp}_{P}^{A}\left(\omega \wedge \widetilde{\Omega}_{A}^{d-i}\right) \subseteq \Omega_{P}^{d}\right\} \cong \operatorname{Hom}_{P}\left(\Omega_{A}^{d-i}, \Omega_{P}^{d}\right),
$$

$i \in \mathbf{Z}$. Obviously we have $\Delta_{A}^{i}=\left\{\omega \in M_{A}^{i}: \omega \wedge \widetilde{\Omega}_{A}^{d-i} \subseteq \Delta_{A}^{d}\right\} \cong \operatorname{Hom}_{A}\left(\Omega_{A}^{d-i}, \Delta_{A}^{d}\right)$. The independence of $\Delta_{A}^{i}, i \in \mathbf{Z}$, from the chosen normalization $P \rightarrow A$ is a consequence of this independence for the case $i=d$, which is proved e.g. in [12]. There is a canonical inclusion $\tilde{\Omega}_{A}^{*} \subseteq \Delta_{A}$. We call $\Delta_{A}^{*}$ the complex of regular differential forms of $\mathbf{A}$. This complex can be constructed (with considerably more effort) for arbitrary (not necessarily reduced) analytic algebras, see [8]. For normal $A$ we have simply $\Delta_{A}^{*} \cong\left(\Omega_{A}^{*}\right)^{* *}$ because in this case both complexes are $A$-reflexive and coincide in codimension $\leqslant 1$. 
In the convergent case the complex $\Gamma_{A}^{*}$ of extendable differential forms may be defined as $\left(\pi_{*} \Omega_{X}^{*}\right)_{x}$, where $\pi: X^{\prime} \rightarrow X$ is a desingularization of the complex space germ $X=(X, x)$ associated to $A$. $\Gamma_{A}^{p}$ is also the module of locally square integrable meromorphic differential forms in degree $p, p \in \mathbf{Z}$ (with the normalization of $A$ as $\Gamma_{A}^{0}$ ).

In the general case one has to replace $X^{\prime}$ by a desingularization of $\operatorname{Spec} A$. In the convergent case both definitions give the same complex. Obviously $\Gamma_{A}^{*}$ can be identified with a subcomplex of $\Delta_{A}^{\circ}$ and is even an algebra (whereas in general $\Delta_{A}^{\circ}$ isn't). Moreover $\Gamma_{A}^{*}=\Gamma_{A^{\prime}}$, where $A^{\prime}$ is the normalization of $A$. For details see [11]. cf. also [4].

(5) Proposition. Let $A \rightarrow B$ be a finite extension of pure-d-dimensional analytic algebras with $a$ well defined rank. Then $\operatorname{Sp}_{A}^{B}\left(\Delta_{B}^{*}\right) \subseteq \Delta_{A}^{*}$ and $\operatorname{Sp}_{A}^{B}\left(\Gamma_{B}^{\cdot}\right)=\Gamma_{A}^{\cdot}$. So there is a canonical diagram

$$
\begin{aligned}
& \Gamma_{A}^{\cdot} \rightarrow \Gamma_{B}^{\cdot} \stackrel{\mathrm{sp}_{A}^{\mathrm{A}}}{\longrightarrow} \Gamma_{A}^{\cdot}
\end{aligned}
$$

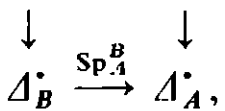

where the composition of the mappings in the lop row is the multiplication with $r:=\operatorname{rank}_{A} B$. If $A$ is normal the inclusion $\tilde{\Omega}_{A}^{\circ} \rightarrow \tilde{\Omega}_{B}^{\cdot}$ extends to an inclusion $\Delta_{A}^{*} \rightarrow \Delta_{B}^{*}$.

Proof. First we consider regular $d$-forms. By definition we have the equalities $\Delta_{B}^{d}=C_{P}^{B} d t_{1} \wedge \ldots \wedge d t_{d}$ and $A_{A}^{d}=C_{P}^{A} d t_{1} \wedge \ldots \wedge d t_{d}$ with a system of parameters $t_{1}, \ldots, t_{d}$ in $A$ and the Dedekind complementary modules $C_{P}^{B}$ resp. $C_{P}^{A}$ associated to the finite extensions $P:=\mathrm{C}\left\langle\left\langle t_{1}, \ldots, t_{d}\right\rangle\right\rangle \rightarrow B$ resp. $P \rightarrow A$.

By the transitivity of the trace $C_{P}^{B}$ is mapped by the trace into $C_{P}^{A}$ and hence $\Delta_{B}^{d}$ into $\Delta_{A}^{d}$. For regular $p$-forms, $p \in \mathbf{Z}$, the assertion follows from the commutative diagram

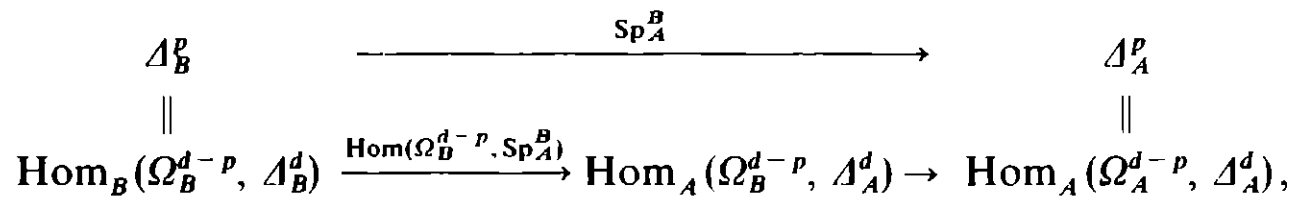

which is obtained from the case $p=d$ just proved. The inclusion $\Delta_{A}^{*} \rightarrow \Delta_{B}^{*}$ is obvious if $A$ and $B$ are both normal. But in general $\Delta_{B^{\prime}} \subseteq A_{B}^{\circ}$, where $B^{\prime}$ is the normalization of $B$.

Now we consider extendable differential forms. For functions the assertion is clear, because the trace of entire elements is again entire.

In the convergent case the homomorphism $A \rightarrow B$ is associated to a finite covering $Y \rightarrow X$ of complex spaces. To prove that for any locally square integrable differential form $\omega$ on $Y$ the trace form $\operatorname{Sp}(\omega)$ is again locally square integrable on $X$ it is sufficient to consider $d$-forms. But outside a suitable exceptional set $S \subseteq X$ (depending on $\omega$ ) the covering $Y \rightarrow X$ is an unramified 
covering $Y^{\prime} \rightarrow X^{\prime}, X^{\prime}:=X-S$, of complex manifolds such that $\omega$ is holomorphic on $Y^{\prime}$ and square integrable. Then it is trivial that the form $\operatorname{Sp}(\omega)$ on $X^{\prime}$ is square integrable too. In the general case let $X:=\operatorname{Spec} A, Y:=\operatorname{Spec} B$ and $\pi$ : $\tilde{X} \rightarrow X$ a desingularization. We consider the commutative diagram

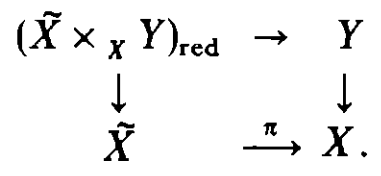

An extendable differential form $\omega$ on $Y$ has an extendable and therefore regular pull-back $\tilde{\omega}$ on $\left(\tilde{X} \times{ }_{X} Y\right)_{\text {red }}$. Hence $\operatorname{Sp}(\tilde{\omega})=\pi^{*} \operatorname{Sp}(\omega)$ is again regular on $\tilde{X}$ and therefore holomorphic on $\tilde{X}$.

(6) Corollary. In the situation of Proposition (5) for $p \in \mathbf{Z}$ the A-module $\Gamma_{A}^{p}$ is a direct summand of $\Gamma_{B}^{p}$, and the vector space $H^{p}\left(\Gamma_{A}^{*}\right)$ is a direct summand of $H^{p}\left(\Gamma_{B}^{*}\right)$. If $A$ is normal $\Delta_{A}^{p}$ is a direct $A$-summand of $\Delta_{B}^{p}$ and $H^{p}\left(\Gamma_{A}^{*}\right)$ a direct summand of $H^{p}\left(\Lambda_{B}^{*}\right)$.

Proof. The assertions follow from the fact that the composition of the complex homomorphism $\Gamma_{A}^{*} \rightarrow \Gamma_{B}^{*}$ and Sp: $\Gamma_{B}^{\cdot} \rightarrow \Gamma_{A}^{*}$ resp. (in the normal case) the composition of $\Delta_{A}^{\circ} \rightarrow \Delta_{B}^{*}$ and $\mathrm{Sp}: \Delta_{B}^{*} \rightarrow \Delta_{A}^{*}$ is the multiplication with $\operatorname{rank}_{A} B$.

As a special case of Corollary (6) let $A$ be normal and $B$ smooth. Then $\Delta_{A}^{p}=\left(\Omega_{A}^{p}\right)^{* *}$ is a Macaulay module for every $p \in \mathbf{Z}$ because it is a direct summand of $\Delta_{B}^{P} \cong B^{\left(\begin{array}{l}d \\ p\end{array}\right)}$. Furthermore $B^{*}=\operatorname{Hom}_{A}(B, A)$ is isomorphic to $B$ as a reflexive $B$-module of rank 1 . Therefore the direct $A$-summand $\left(\Omega_{A}^{p}\right)^{*}=\left(\Omega_{A}^{p}\right)^{* * *}$ of $B^{*\left(\begin{array}{l}d \\ p\end{array}\right)} \cong B^{\left(\begin{array}{c}d \\ p\end{array}\right)}$ is a Macaulay module too. As a consequence one gets for example $\operatorname{Ext}_{A}^{i}\left(\Omega_{A}^{p}, A\right)=0$ for all $i=1, \ldots, s-2, s:=$ co$\operatorname{dim}(\operatorname{sing} A)$ (and especially the rigidity of $A$ if $s \geqslant 3$ ), cf. [1], 16.E and [17].

(7) Corollary. Let $A \rightarrow B$ be a nondegenerate extension of analytic algebras (i.e. $\operatorname{dim} B=\operatorname{dim} A+\operatorname{dim} B / \mathrm{m}_{A} B$ ) with $A$ normal and $B$ reduced and pure dimensional. If $\Gamma_{B}^{p}=\Delta \Delta_{B}^{p}$ for some $p \in \mathbf{Z}$ then also $\Gamma_{A}^{p}=\Delta_{A}^{p}$.

Proof. If the elements $f_{1}, \ldots, f_{m} \in B$ form a system of parameters in the fibre $B / \mathrm{m}_{A} B$ the algebra $B$ is a finite extension of $A^{\prime}:=A\left\langle\left\langle f_{1}, \ldots, f_{m}\right\rangle\right\rangle$. By (5) we have $\Gamma_{A^{\prime}}^{p}=\operatorname{Sp}_{A^{\prime}}^{B}\left(\Gamma_{B}^{p}\right)=\operatorname{Sp}_{A^{\prime}}^{B}\left(\Delta_{B}^{p}\right)=\Delta_{A^{\prime}}^{p}$. Because of the formula

$$
\Delta_{A^{\prime}}^{p}=\Delta_{A}^{p}\left\langle\left\langle f_{1}, \ldots, f_{m}\right\rangle\right\rangle \oplus \sum_{i} d f_{i} \wedge \Delta_{A}^{p^{-1}}
$$

and the analogous one for $\Gamma_{A}^{p}$, we get $\Gamma_{A}^{p}=\Delta_{A}^{p}$.

We remark that the equality $\Gamma_{B}^{p}=\Delta D_{B}$ always holds if $p \leqslant$ $\operatorname{codim}(\operatorname{sing} B)-2$, cf. [3].

(8) Corollary. Let $A \rightarrow B$ be a nondegenerate extension of normal analytic algebras with $\Gamma_{B}^{1}=\Delta_{B}^{1}$. If $\Delta_{A}^{1}\left(=\left(\Omega_{A}^{1}\right)^{* *}\right)$ is free then $A$ is smooth. 
Proof. By (7) $\Gamma_{A}^{1}=\Delta_{A}^{1}$ is free. The result now follows from the following criterion for smoothness: If $A$ is reduced and pure dimensional and if $\Gamma_{A}^{1}$ is free as an $A$-module then $A$ is smooth, cf. [10], [11].

Corollary (8) is a partial generalization of a result in [19], where $\Omega_{B}^{1}$ is assumed to be reflexive.

(9) Corollary. For a quasihomogeneous normal analytic algebra $B$ the canonical homomorphism

$$
\Omega_{B}^{1} / \mathrm{m}_{B} \Omega_{B}^{1} \rightarrow \Delta_{B}^{1} / \mathrm{m}_{B} \Delta_{B}^{1}
$$

is injective.

Proof. Let $f_{1}, \ldots, f_{d}$ be a homogeneous system of parameters in $B$ and $A$ the algebra $\mathbf{C}\left\langle\left\langle f_{1}, \ldots, f_{d}\right\rangle\right\rangle \subseteq B$. By $\delta$ we denote the Euler derivations and the corresponding linear forms on the modules of differentials too. The diagram

$$
\begin{aligned}
& \Omega_{A}^{1} \rightarrow \Omega_{B}^{1} \rightarrow \Delta_{B}^{1} \stackrel{\text { sp }}{\rightarrow} \Omega_{A}^{1} \\
& \delta \downarrow \\
& \delta \downarrow \\
& A
\end{aligned}
$$

is commutative with $\delta\left(\Omega_{B}^{1}\right)=\mathfrak{m}_{B}$. So $\delta$ induces an isomorphism $\delta$ : $\Omega_{B}^{1} / \mathfrak{m}_{B} \Omega_{B}^{1} \rightarrow \mathfrak{m}_{B} / \mathfrak{m}_{B}^{2}$. The $B$-linear form $\delta: \Delta_{B}^{1} \rightarrow B$ isn't surjective, otherwise Spo $\delta=\delta \circ S p$ and hence $\delta: \Omega_{A}^{1} \rightarrow A$ would be surjective too. Therefore $\delta$ induces a homomorphism $\delta: \Lambda_{A}^{1} / \mathrm{m}_{B} \Delta_{B}^{1} \rightarrow \mathrm{m}_{B} / \mathrm{m}_{B}^{2}$. The commutative diagram

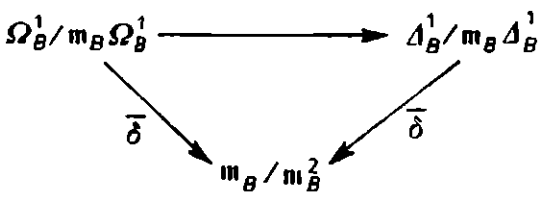

gives now the assertion.

(10) Corollary (Hochster [6]). Let $B$ be a quasi-homogeneous normal analytic algebra. If $\Delta_{B}^{1}\left(=\left(\Omega_{B}^{1}\right)^{*}\right)$ is free then $B$ is smooth.

Proof. By (9) the minimal number of generators of the $B$-module $\Omega_{B}^{1}$ is at most the minimal number of generators of $\Delta_{B}^{1}$. By assumption this is $d:=\operatorname{dim} B$. But $\Omega_{B}^{1}$ is a module of rank $d$, so it is free.

Corollary (9) answers a question raised by E. Platte in [20], Rem. 2.5. A further proof of $(10)$ is given in [16].

(11) Nonramification. Related to the considerations above is the question whether for a finite extension $A \rightarrow B$ the property of being unramified can be characterized with the help of regular or extendable differentials instead by using Kähler differentials. More precisely: Let $A \rightarrow B$ be a finite extension of normal analytic algebras. By a classical result (which we have used before) the equality $A=B$ holds if and only if the canonical homomorphism 
$B \otimes_{A} \Omega_{A}^{1} \rightarrow \Omega_{B}^{1}$ is surjective. Is it possible to replace $\left(\Omega_{A}^{1}, \Omega_{B}^{1}\right)$ by $\left(\Delta_{A}^{1}, \Delta_{B}^{1}\right)$ or $\left(\Gamma_{A}^{1}, \Gamma_{B}^{1}\right)$ ? In general this seems to be a difficult problem. The surjectivity of $B \otimes_{A} \Delta_{A}^{1} \rightarrow \Delta_{B}^{1}$ or $B \otimes_{A} \Gamma_{A}^{1} \rightarrow \Gamma_{B}^{1}$ implies that the extension $A \rightarrow B$ is unramified in codimension 1 , because the modules $\Delta_{A}^{1}, \Gamma_{A}^{1}$ and $\Omega_{A}^{1}$ resp. $\Delta_{A}^{1}, \Gamma_{B}^{1}$ and $\Omega_{B}^{1}$ coincide for the nonsingular locus of $A$ resp. $B$. Let us assume that $\Delta_{A}^{1}$ is a free $A$-module. Then conversely the mapping $B \otimes_{A} \Delta_{A}^{1} \rightarrow \Delta_{B}^{1}$ is surjective if the extension $A \rightarrow B$ is unramified in codimension 1 because then the two reflexive $B$-modules $B \otimes_{A} A_{A}^{1}$ and $A_{B}^{1}$ coincide in codimension 1 . A positive answer to our question would imply that $A$ has no strict extensions unramified in codimension 1 (if $\Delta_{A}^{1}$ is free). So in the case $\operatorname{dim} A=2$ the algebra $A$ would be pure and hence smooth using a result of Flenner [2]. This would solve the Zariski-Lipman problem in dimension 2 .

Here we prove:

(12) Let $A \rightarrow B$ be a finite extension of normal analytic algebras. Then we have:

(i) If $B \otimes_{A} \Delta_{A}^{1} \rightarrow \Delta_{B}^{1}$ (resp. $\left.B \otimes_{A} \Gamma_{A}^{1} \rightarrow \Gamma_{B}^{1}\right)$ is surjective and if

$$
\left.\Omega_{B}^{1} / m_{B} \Omega_{B}^{1} \rightarrow \Delta_{B}^{1} / m_{B} \Delta_{B}^{1} \quad \text { (resp. } \Omega_{B}^{1} / m_{B} \Omega_{B}^{1} \rightarrow \Gamma_{B}^{1} / m_{B} \Gamma_{B}^{1}\right)
$$

is injective (which by (9) holds if $B$ is quasihomogeneous) then $A=B$.

(ii) If $B \otimes_{B} \Delta_{A}^{1} \rightarrow \Delta_{B}^{1} \quad\left(\right.$ resp. $\left.B \otimes_{A} \Gamma_{A}^{1} \rightarrow \Gamma_{B}^{1}\right)$ is surjective and if $A$ is quasihomogeneous then $A=B$.

Proof. We treat the case of regular differential forms. The proof for extendable forms runs along the same lines. From the surjectivity of $B \otimes \Delta_{A}^{1} \rightarrow \Delta_{B}^{1}$ one deduces the surjectivity of $\Delta_{A}^{1} / \mathrm{m}_{A} \Delta_{A}^{1} \rightarrow \Delta_{B}^{1} / \mathrm{m}_{B} \Delta_{B}^{1}$ and the equality $\mathrm{m}_{B} \Delta_{B}^{1}=\mathrm{m}_{B} \Delta_{A}^{1}$. Because of $\mathrm{Sp}\left(\mathrm{m}_{B}\right)=\mathrm{m}_{A}$ the trace induces by Theorem (5) a mapping $\Delta_{B}^{1} / \mathrm{m}_{B} \Delta_{B}^{1} \rightarrow \Delta_{A}^{1} / \mathrm{m}_{A} \Delta_{A}^{1}$ which is up to the factor rank $_{A} B$ left inverse to the first one and therefore invertible.

To prove (i) we consider the commutative diagram

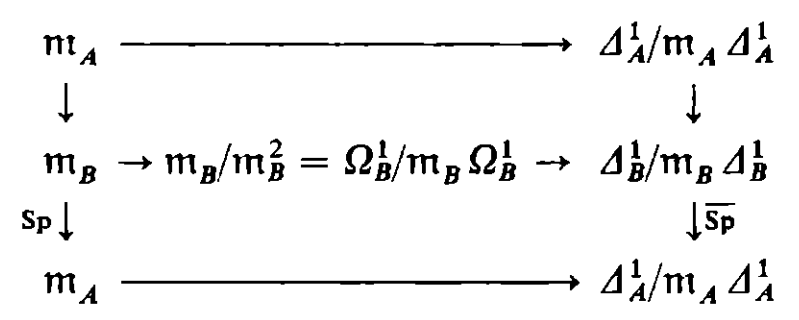

and get kern $\mathrm{Sp} \subseteq \mathrm{m}_{B}^{2}$. It follows $\mathrm{m}_{B}=\mathrm{m}_{A}+\operatorname{kern} \mathrm{Sp}=\mathrm{m}_{A} B+\mathrm{m}_{B}^{2}$ and $\mathrm{m}_{B}=\mathrm{m}_{A} B$, i.e. $A=B$.

The case (ii) can be reduced to the case (i): Without loss of generality we may assume that $A$ and $B$ are complete. The Euler derivation $\delta$ of $A$ can be extended to a derivation of $B$ because the $A$-linear form $\Delta_{A}^{1} \rightarrow A$ 
corresponding to $\delta$ defines a $B$-linear form on $\Delta_{B}^{1}=\left(B \otimes_{A} \Delta_{A}^{1}\right) / \mathrm{t}\left(B \otimes_{A} \Delta_{A}^{1}\right)$. (The extendability of $\delta$ follows already from the property that the extension $A \rightarrow B$ is unramified in codimension 1.) The result (2.14) in [25] implies that $B$ is quasihomogeneous, and part (i) can be applied.

\section{References}

[1] W. Bruns and U. Vetter, Determinantal Rings, Lecture Notes in Math. 1327 (1988).

[2] H. Flenner, Reine lokale Ringe der Dimension zwei, Math. Ann. 216 (1975), 253-263.

[3] -, Extendability of differential forms on non isolated singularities, Invent. Math. 94 (1988), 317-326.

[4] Ph. Griffiths, Variations on a theorem of Abel, Invent. Math. 35 (1976), 321-390.

[5] R. Hartshorne, On the De Rham cohomology of algebraic varieties, Publ. Math. IHES 45, (1976), 5-99.

[6] M. Hochster, The Zariski-Lipman conjecture in the graded case, J. Algebra 47 (1977), 411-424.

[7] R. Hübl, Spuren von Differentialformen und Hochschild-Homologie, Dissertation, Regensburg 1987.

[8] M. Kersken, Residuenkomplex in der lokalen algebraischen und analytischen Geometrie, Math. Ann. 265 (1983), 423-455.

[9] -, Reguläre Differentialformen, Manuscripta Math. 46 (1984), 1-25.

[10] -, Ein Regularitätskriterium für analytische Algebren, Arch. Math. 51 (1988), 434-439.

[11] -, Differentialformen in der algebraischen und analytischen Geometrie, Habilitationsschrift, Bochum 1987.

[12] E. Kunz, Holomorphe Differentialformen auf algebraischen Varietäten mit Singularitäten I, Manuscripta Math. 15 (975), 91-108.

[13] -, Kähler Differentials, Braunschweig/Wiesbaden 1986.

[14] J. Lipm a n, Free derivation modules on algebraic varieties, Amer. J. Math. 87 (1965), 874-898.

[15] -, Residues and Traces of Differential Forms via Hochschild Homology, Contemp. Math. 61, 1986.

[16] E. Platte, Ein elementarer Beweis des Zariski-Lipman-Problems für graduierte analytische Algebren, Arch. Math. 31 (1978), 143-146.

[17] -, Pure descent for the module of Zariski differentials, Proc. Amer. Math. Soc. 82 (1981), 7-12.

[18] -, Jacobi-Algebra und Blätterzahl von analytisch-verzweigten Überlagerungen isolierter Hyperflächensingularitäten, Arch. Math. 39 (1982), 121-125.

[19] -, Nicht-ausgeartete reguläre Überlagerungen, Math. Z. 180 (1982), 413-417.

[20] -, The module of Zariski-differentials of a normal graded Gorenstein-singularity, J. Pure Appl. Algebra 30 (1983), 301-308.

[21] -, Differentielle Methoden in der Lokalen Algebra, Osnabrücker mathematische Schriften, 1988.

[22] G. Scheja, Über ganz-algebraische Abhängigkeit in der Idealtheorie, Comment. Math. Helv. 45 (1970), 384-390.

[23] G. Scheja und U. Storch, Differentielle Eigenschaften der Lokalisierungen analytischer Algebren, Math. Ann. 197 (1972), 137-170.

[24] -, -, Über Spurfunktionen bei vollständigen Durchschnitten, J. Reine Angew. Math. 278/279 (1975), 174-190.

[25] G. Scheja and H. Wie be, Zur Chevalley-Zerlegung von Derivationen, Manuscripta Math. 33 (1980), 159-176. 